\title{
Recognition of domain patterns using high-resolution single crystal X-ray diffraction
}

\author{
Semën Gorfman
}

\author{
Tel Aviv University, Tel Aviv, Israel
}

gorfman@tauex.tau.ac.il

Ferroelectric / ferroelastic / ferroic domains are the volumes of a material where polarization / strain / any other order parameter are uniform. Domain patterns usually appear as a result of a structural phase transition between para and ferro-electric phases, which changes the symmetry (including crystal system and the space group type). Domain patterns play the central role for the materials properties, e.g. domain-wall motion may majorly contribute to the enhancement of piezoelectric effect and dielectric permittivity [1]. The knowledge of key parameters of domain patterns (such as orientation / mobility of domain walls, volume fraction of specific domains) can help to characterize, predict and even tailor the properties of ferroelectric materials.

Domain patterns may be extremely complicated, especially for a low symmetry material. Unfortunately, only a handful of experimental techniques are suitable for characterization of domain patterns directly. None of them are powerful enough to image domains in three-dimensional bulk volumes of a material and fast enough to probe their response to alternating external perturbation (e.g. temperature or electric field). Although, high-resolution X-ray diffraction is clearly capable to fill this gap, it still has to be significantly advanced and improved to be efficient and reliable. The geometry of single crystal X-ray diffraction pattern from a multidomain crystal is as complex as domain patterns themselves. It might be very hard to recognize and observe individual domains types / domain walls in the bulk, especially in the case of monoclinic symmetry where the number of domains and domain walls between them is very large.

The morphology of domain patterns are governed by the lost symmetry elements and conditions of mechanical compatibility, which were found by Fousek, Vanocek [2] and later by Sapriel [3].

The goal of this work is to re-formulate the conditions of mechanical compatibility in the form that it is suitable for the analysis of split peaks in high-resolution X-ray diffraction. We inspect diffraction from multi-domain ferroelectric crystals accordingly and show the way to assign different peak components to the individual domains.

The experimental part of the work is done at the single crystal four-circle X-ray diffractometer in Tel Aviv University (Tel Aviv, Israel) and Swiss-Norwegian Beamlines at the European Synchrotron (Grenoble, France). We used the methodology outlined in [4] to accumulate three-dimension X-ray diffraction intensity distribution in the reciprocal space around split Bragg reflection diffracted from the domains in $\mathrm{BaTiO}_{3}(\mathrm{BT})$ and $\mathrm{Na}_{0.5} \mathrm{Bi}_{0.5} \mathrm{TiO}_{3}$ (NBT). The positions of individual sub-peaks in the reciprocal space can be marked and geometrical parameters of the peak groups (e.g. the inter-peak vectors) can be analyzed.

The theoretical part of this work involved the geometrical analysis of domain walls of different symmetry, where a "wall" is defined as a strain-free planar interface between the arbitrary pair of domains. We developed the algorithm and MATLAB-based computer program which predicts the list of mechanically compatible domains / orientation of the domain walls, angle between polarization vectors (if the direction of polarization vector is known) and the matrix of orientation of one domain relative to another.

Furthermore, we proved (analytically) that every pair of mechanically compatible domains produces a pair of Bragg sub-peaks, separated in the reciprocal space along the direction that is perpendicular to the domain wall. This remarkable result is used to recognize possible pairs of domains in the diffraction pattern directly.

We demonstrate such recognition for the simple case of tetragonal domains in BT crystal and use to investigate the formation of the domains in the course of the phase transition to the lower symmetry monoclinic phases in PZT and NBT.

[1] Damjanovic, D. Reports on Progress in Physics, (1999), 61(9), p. 1267, 1999.

[2] Fousek, J., Vanocek, V. J. Appl. Phys., (1969), 40(135), 135-142.

[3] J. Sapriel, "Domain-wall orientations in ferroelastics", Physical Review B12, p. 5125, 1975. 1974

[4] Gorfman, S., Choe, H., Zhang, G., Zhang, N., Yokota, H., Glazer, A.M., Xie, Y., Dyadkin, V., Chernyshov, D., Ye Z.-G. J. Appl. Cryst. (2020), 53(4), 1039-1050.

Keywords: ferroelectric perovskites, ferroelastic domains, X-ray diffraction 